\title{
Positioning Usability in an Organisation: Can usability really help organisations achieve their business objectives?
}

\author{
Janice Anne Rohn, Manager \\ Usability Labs and Services \\ Sun Microsystems \\ 2550 Garcia Avenue \\ Mountain View, CA 94043-1100 \\ USA \\ rohn@sandybeaches.eng.sun.com
}

\author{
Sarah Bloomer, Director \\ The Hiser Group \\ P.O. Box 312 \\ Prahran, Victoria 3181 \\ AUSTRALIA \\ sarahb@mel.hiser.com.au
}

\section{TOPIC}

This panel addresses the question of whether usability can help organisations to achieve their business goals through the integration of usability methods during the design and development of software.

Usability is beginning to be sold to managers at a strategic level with the promise that it can impact and support business goals and initiatives: costjustification figures seem to indicate that usability really can impact the bottom line, whether in improving customer service and satisfaction or creating a greater market share for a particular product. But can usability actually fulfil this promise? And what is preventing usability from being as readily accepted as other strategic initiatives such as change management, business process re-engineering or training initiatives?

Our aim is to take a critical look at usability and the benefits that it claims by bringing together professionals who include usability in their strategies. We will compare usability to other techniques which aim to make organisations more competitive, reduce costs or enhance productivity, in order to gain an understanding of how usability complements or conflicts with these techniques and thereby deliver business benefits.

\section{PANEL MEMBERS}

Panel members consist of practitioners from areas related to usability, specifically, business process reengineering, change management, training, and software development, as well as a discussant familiar with introducing usability into organisations. In the spirit of Interact's international focus, we have attempted to bring together panelists from a range of organisations, professions as well as continents to draw together a broad spectrum of experiences.

The positions focus on the value of usability and experiences of whether the benefits (such as reduced training costs, enhanced productivity, greater user acceptance, and reduced development time) can actually be realised. They will provide hard evidence of the success of their own techniques, such as statistics, testimonials and case studies. The following questions will be considered:

1. How is usability positioned in your organisation, and does it vary from organisation to organisation?

2. Can usability deliver significant business benefits that support an organisation in becoming more competitive?

3. How does usability complement or conflict with other methods and techniques designed to enhance the competitive advantage?

4. How can the usability practitioner achieve buyin for usability across an organisation, from end-users to developers to upper management?

\section{Usability Manager - Janice Rohn, Sun Microsystems, USA}

The usability manager is often tasked with the job of convincing upper management, project leaders and often end-users (in the case of in-house software development) of the benefits and reasoning for allocating resources to usability. This can be 
particularly challenging in technology companies given the emphasis on technology-driven products and increasingly shortened development cycles. With an increasing emphasis on internet products, there is a tension between just getting a product out versus taking some initial time to define users, requirements, and usable design. Usability can only succeed by demonstrating the importance of performing these activities and by keeping pace with accelerated development schedules.

Janice Rohn is Manager of Usability Labs and Services at Sun Microsystems. Janice joined Sun in 1992, when she founded the usability group and designed and built usability labs for Suns sites in California and Colorado. Janice has been working on human-computer interaction and strategy issues across Sun, and has led several efforts to improve the integration and usage of $\mathrm{HCI}$ methods. Janice is President of the Usability Professionals Association. Prior to joining Sun, Janice worked at Apple on the usability and design of products such as System 7, QuickTime, the PowerBooks, and the Quadras. Prior to Apple, Janice was a research assistant at Stanford University in expert systems and medical informatics. Janice has a number of publications including a chapter in Cost Justifying Usability.

\section{Business Process Re-Engineering - Mark Andrew, ALARA, Australia}

Business process re-engineering is becoming increasingly popular in the current climate of downsizing and restructuring. Changes to business processes are often facilitated through technology since new processes require new systems. This puts usability practitioners in a good position to have an impact on new business processes.

Usability is commonly defined as an outcome during these assignments, rather than as a consulting effort. It is sometimes recognised by clients as a performance indicator for employees (i.e. are the people and the work "demonstrating usability"?) but rarely recognised as a progressive journey. Consequently, a consulting culture of "trojan horse usability" has developed (i.e. ensure usability by subterfuge first, then reveal the process). Along the way, both the client's, and consultant's "culture" changes as a result of such partnering efforts. Mark will be in a position to talk about such experiences.

Mark Andrew works with industrial clients in transport, mining and manufacturing. He aims to help them understand their operational risks, in order to improve business process efficiency. Assignments usually involve task analysis and work methods design, and result in redesigned equipment, procedures and communication. Before returning to ALARA to head up their Victorian office in 1995, Mark consulted in business process re-engineering and strategic management.

\section{Change Management - Dianne Saker,} Andersens Consulting, Australia \& USA

Usability as a discipline, and if implemented well, can impact the business bottom line, not just for IT development methodologies, but for entire user communities and the organisation as a whole. Usability demands a paradigm shift in behaviour, work processes and people change. A usability practitioner must effectively understand broad change management principles and the weighting of usability in managing small or large scale change. To convince upper management to commit resources, money and time to usability requires an understanding of such change and the pressures of large scale implementation.

Dianne Saker is a Change Management Manager for Andersen Consulting and leads the Australian Usability team for her practice. Dianne has pioneered usability and $\mathrm{HCl}$ methods as a discipline to a number of key Australian organisations in the Financial Services and Government industries. Dianne is a global usability expert and has consulted to organisations around the world on building business need for usability and establishing usability disciplines into large technology, process and people change projects.

\section{Client Management - Malcolm Keys, Optus Communications, Australia}

Improving job performance while reducing training costs is the goal of performance support initiatives. Usability and user interface design complement this goal by providing practical methods for improving computer systems. However, for usability to deliver benefits, it must be integrated into the development life cycle and culture. When it is not integrated into the mainstream practices of the organisation, usability appears to provide only bad news at the wrong time, and is therefore often ignored.

At Optus, usability has been introduced through the training and performance improvement initiative and is therefore inherently cross-disciplinary. Our most successful activity has been to measure the usability of each of our systems as they are delivered and publicise the results within the organisation. This is leading to culture which places a high importance on usability of its products and makes use of the available usability infrastructure.

Malcolm Keys is a Usability Manager with the Optus Communications Systems Training where he leads the usability initiative. Since joining Optus, Malcolm has established a set of usability techniques tools designed to be used by systems development teams. Prior to joining Optus, Malcolm worked as a senior usability consultant at The Hiser Group and was an independent consultant in multimedia.

\section{Discussant - Randolph Bias, BMC Software, USA}

Randolph Bias is Manager of Usability Engineering for BMC Software, Inc. He has a Ph.D. in human experimental psychology from the University of Texas at Austin, and 15 years' experience in software usability engineering at Bell Labs and IBM. Randolph is a "Certified Human Factors Professional" and is co-editor of CostJustifying Usability_(R. G. Bias \& D. J. Mayhew, Eds., Academic Press: Boston, 1994). 


\section{Format For Running The Panel}

A panel format was selected for this topic in order to explore various viewpoints regarding the impact of usability at the corporate level, providing an opportunity to explore these viewpoints, and resulting in a stimulating debate about whether usability can have an impact on business objectives. The panel format facilitates the exchange of practical knowledge between panelists and audience members.

This is a comparative panel. Although it doesn't directly compare approaches and techniques within an area, it does look at the approaches between areas, and how each area potentially influences each other, positively or negatively. The common ground in this panel is the passing of knowledge between several groups of practitioners who may impact each other on a strategic project.
This panel will aim to be highly interactive. The audience will be asked to submit their questions on cards which are handed out when they arrive. The concept of organisational usability will be briefly presented (5 minutes) by one of the panel organisers. To open the debate, each panelist will give a short presentation ( 5 minutes) of their view of how usability supports or conflicts with their business orientation or area of expertise. The discussant will then summarise the four positions ( 5 minutes).

The second half hour will focus on a set of predefined questions prepared to focus the discussion on strategic questions. An overhead projector will be used to capture key issues raised by each panelist. Input from the audience will be encouraged. The last half hour will address questions from the audience as submitted by the cards. Throughout the session, we will capture things that work and things that don't work from the panelists and the audience, thereby building up a total picture of the debate as it unfolds. 